\title{
Similarities and differences in microwave and optical radiation detection
}

Negussie Tirfessa, Chandrasekhar Roychoudhuri

Negussie Tirfessa, Chandrasekhar Roychoudhuri, "Similarities and differences in microwave and optical radiation detection," Proc. SPIE 11143, Fifteenth Conference on Education and Training in Optics and Photonics: ETOP 2019, 111433W (2 July 2019); doi: 10.1117/12.2525442 Photonics: ETOP 2019, 2019, Quebec City, Quebec, Canada 


\title{
Similarities and differences in microwave and optical radiation detection
}

\author{
Negussie Tirfessa' and Chandrasekhar Roychoudhuri ${ }^{2}$ \\ 'Department of Physical Sciences, Manchester Comm. College, Manchester, CT, USA \\ ${ }^{2}$ Physics Department, University of Connecticut ${ }^{2}$ Storrs, CT, USA \\ 'ntirfessa@manchestercc.edu, ${ }^{2}$ Chandra.Roychoudhri@uconn.edu
}

\begin{abstract}
The differences and similarities between the detection processes involved in the microwave and visible electromagnetic (EM) waves are presented for the case of two beam superposition. A microwave receiver measures the motion of conduction electrons which responds linearly to the superposed time dependent EM field amplitudes. A visible light detector also responds to the superposition of the EM field amplitudes due to interactions with the detecting dipoles. However, the visible light detector measures the quantum of energy absorbed and responds to the square modulus of the superposition of the time dependent amplitudes of the EM fields. The measured current in the microwave detector is directly proportional to the superposed microwave amplitudes. The measured energy in visible light detector is directly proportional to the square modulus of the superposed visible light amplitudes. We will highlight the similarities and differences in the detection processes. These important similarities and difference are not underscored in most optics textbooks.
\end{abstract}

Key Words: Electromagnetic Field, Microwave, Visible Light, Superposition of Microwaves, Superposition of Visible Light, Quantum Detector versus Classical Detector.

\section{INTRODUCTION}

Our breakthrough understanding of the electromagnetic (EM) wave came after James Clerk Maxwell in 1864[1] put together the field equations and predicted the existence of EM waves. The process of generating and receiving/detecting EM waves was first demonstrated by German physicist Heinrich Rudolf Hertz in 1888[2, 3]. Hertz's great experiments on low frequency EM waves (radio waves) confirmed Maxwell's EM theory and predictions. This critical work is what also established the EM field existence as a perpetual wave independently traveling through space. The EM waves range from the smallest frequencies of radio waves (then to microwaves, infrared, visible, ultraviolet, x-rays) to the highest frequencies of gamma rays. The human eye is capable of detecting only less than $1 \%$ of the vast frequency ranges of the EM waves generated in our universe. Our major discoveries of the universe that have been improving our understanding are heavily dependent on the EM waves we detect and interpret by using various detectors. These detectors range from radio receivers to gamma ray detectors. The detection process involves the interaction of EM waves with electrons (bound or relatively free), atoms, and dipoles. Our understanding of the physical process depends on the information we get from the detectors. It is only when the EM waves interact with matter that we are able make measurements. The detectors act as mediators for mapping our understanding of the interaction process between the EM waves and matter. The interaction of EM waves with each other in free space with no detectors would be a plausible prediction with no measurement data. There are no rainbows observable in the sky in the absence of spherical water droplets.

Current understanding of the origin and evolution process of the universe is the result of our ability to detect EM waves at various frequencies. The interstellar gas cloud and dust, the temperature of stars, and the recent discoveries of gravitational waves that used laser interferometry, the discoveries of new particles at various particle accelerators around the world, the recently released image of black hole are all results obtained through detectors. The recently published image of a supper massive black hole [2] at the center of a distant galaxy -Messier 87 shows radiation measurement of a

Fifteenth Conference on Education and Training in Optics and Photonics: ETOP 2019, edited by

Anne-Sophie Poulin-Girard, Joseph A. Shaw, Proc. of SPIE Vol. 11143, 111433W · ( 2019

SPIE, ICO, IEEE, OSA · CCC code: 0277-786X/19/\$18 · doi: 10.1117/12.2525442

Proc. of SPIE Vol. $11143111433 \mathrm{~W}-1$ 
gas around the black hole. The black hole is estimated to be about 55 million light years away from Earth. We are able to assign this radiation from matter at such large distance inherently implies that the EM waves we receive are not affected by other radiations generated from other stars and gases in the intermediate space. The subject of non-interaction of EM waves with each other is a gap in the process that needs to be studied. For an undergraduate students studying electromagnetism and developing problem solving skills, the emphasis on the physical interaction process between EM wave and matter makes a rich learning experience. The information we gather through the eyes of detectors plays an important role in future discoveries and in constantly advancing our knowledge about the small and large objects in the universe.

The purpose of this work is to highlight the difference in the detection process between the microwave detectors and optical detectors. We believe addressing and emphasizing the process enhances the optics education at the undergraduate level and gives students a component of the learning process that is often not addressed.

\section{SUPERPOSITION OF WAVES}

The superposition principle in classical physics allows as to treat solutions of the wave equations for waves traveling on a string

$$
\frac{\partial^{2} S}{\partial x^{2}}=\frac{1}{\mathrm{v}^{2}} \frac{\partial^{2} S}{\partial t^{2}}
$$

Where $S=S(x, t)$ is the displacement of a string element of mass located at position $\mathrm{x}$ as a function time and $\mathrm{v}$ is the speed of the wave. A standard sinusoidal solution in the form of $S=S_{o} \cos \left(\frac{2 \pi}{\lambda}(x-\mathrm{v} t)\right)$ would satisfy the wave equation. According to the superposition principle, a linear combinations of such solutions would also satisfy the equation [5]. The physical process behind the principle tells us that the string elements respond to the net displacements arising from the superposed disturbances. We may write a similar wave equation for a charge-free electromagnetic waves in free space propagating along the $\mathrm{x}$-axis

$$
\frac{\partial^{2} E}{\partial x^{2}}=\frac{1}{\mathrm{v}^{2}} \frac{\partial^{2} E}{\partial t^{2}}
$$

Where here $E=E(x, t)$ is the electric field amplitude along the $\mathrm{y}$-axis and $\mathrm{v}=c=1 / \sqrt{\varepsilon_{0} \mu_{0}}=3 \times 10^{8} \mathrm{~m} / \mathrm{s}$ is the speed of light in free space. A sinusoidal electric field solution of the form $E_{1}=E_{0} \cos (k x-2 \pi v t)$ satisfies the equation. Superposition of two or more waves also satisfies the solutions. In the absence of detectors in free- space, the superposition would not be observable. In the presence of detecting dipoles, the superposition of the fields would be performed through the interaction between the incident electric field(s) and the detector (electron, atom, molecules and dipole). The interaction results in a transfer of energy from the incident field to the detecting matter. In the case of low frequency EM waves (radio and microwave) the detectors can be conduction electron in the antennas that induce electric current in the LRC circuit tuned to the frequency of the incoming EM wave. The induced current oscillates with amplitude and frequency proportional to the incident EM wave amplitude and frequency. In the case of visible EM waves, the detecting dipoles absorb energy proportional to the square of the amplitude of incident EM wave.

\section{MICROWAVE DETECTION}

Hertz's EM wave detection experiment is also considered as a start of antenna. Microwave generation and transmissions were further developed during World War II [7]. Microwaves have an approximate range of $0.1-300 \mathrm{GHz}$ [5]. In the detection process of microwave fields, the electric field interact with conduction electrons in the antenna. This in turn induces similar oscillations in the conduction currents of the LRC circuit resulting in a measurable AC current. Let us consider an EM wave received by the antenna dipoles to be of the form $E=E_{0} \cos (2 \pi v t)$. The induced current I is also proportional to this electric field with some phase difference and will have the form of $I=I_{0} \cos (2 \pi v t+\phi)$ where 
$I_{0}$ is the current amplitude and $\phi$ is the phase difference. The receiving dipole oscillates with the same frequency as the electric field of the EM wave (see Figure 1). The LCR circuit detecting the wave will have a resonant frequency that matches the frequency $v=1 / \sqrt{L C}$.

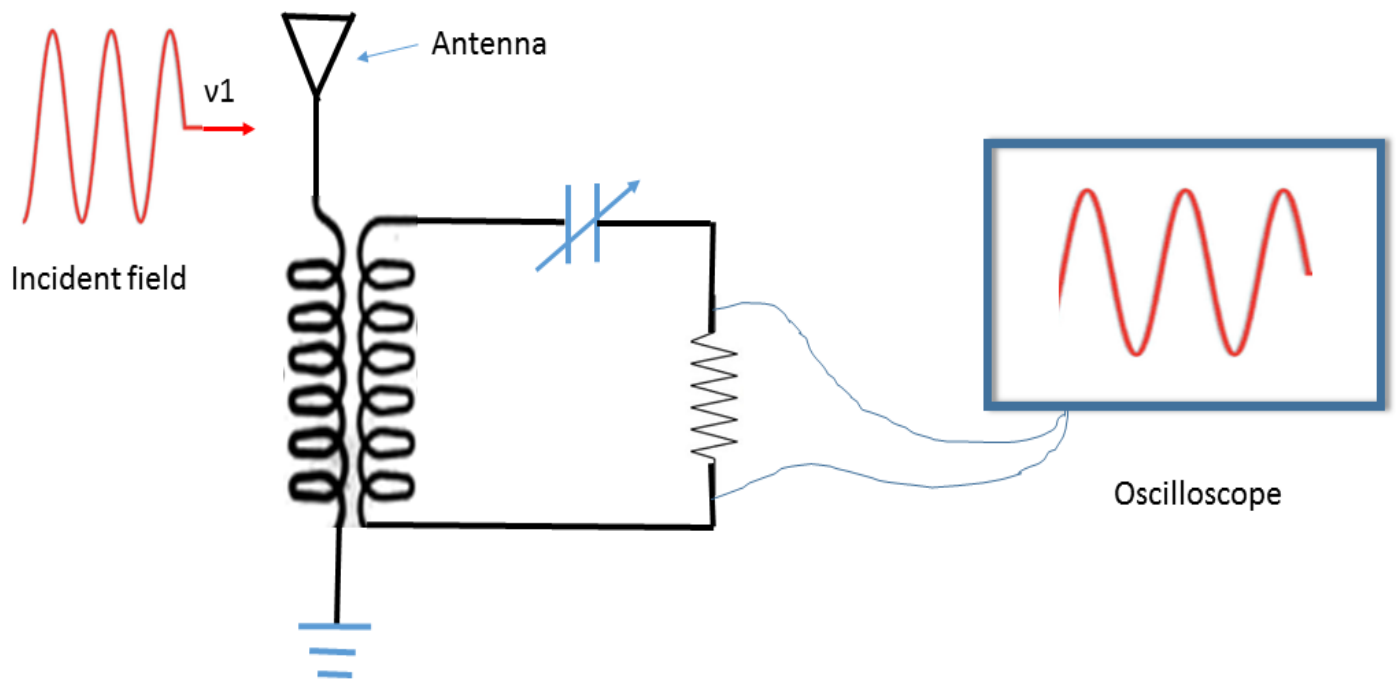

Figure 1. A microwave electric field is incident on an antenna that sets the conduction electrons in an oscillatory motion with the same frequency as the incident electric field. This induces an AC current in the detecting LRC circuit that is displayed on an oscilloscope.

The energy carried by the EM wave is proportional to the square of electric field amplitude. In the case of detector antenna, there is no quantum transition between energy levels of the electrons in the conductor. Instead, the electrons in the conduction band respond to the external EM field by oscillating with the same frequency.

\section{SUPERPOSITION OF MICROWAVES}

The superposition of two microwave fields of the same amplitude and frequency of the form $E_{1}=E_{0} \cos (2 \pi v t)$ and $E_{2}=E_{0} \cos (2 \pi v t+\phi)$ would give $E=2 E_{0} \cos (\phi / 2) \cos (2 \pi v t+\phi / 2)$. The amplitude of the resulting electric field depends on their phase difference as can be seen on a standing wave that can be generated and detected. Depending on the phase difference.

For the superposition of microwaves of different frequencies, we consider the following electric field vectors

$$
E_{1}=E_{0} \cos \left(2 \pi v_{1} t\right) \text { and } E_{2}=E_{0} \cos \left(2 \pi v_{2} t\right)
$$

The combined electric field experienced by the conduction electrons in the detecting antenna would be

$$
E=E_{1}+E_{2}=2 E_{0} \cos (2 \pi \bar{v} t) \cos \left(2 \pi v_{b} t\right)
$$

Where $\bar{v}=\frac{1}{2}\left(v_{1}+v_{2}\right)$ is the mean frequency and $v_{b}=\frac{1}{2}\left|v_{1}-v_{2}\right|$ is the beat frequency. 


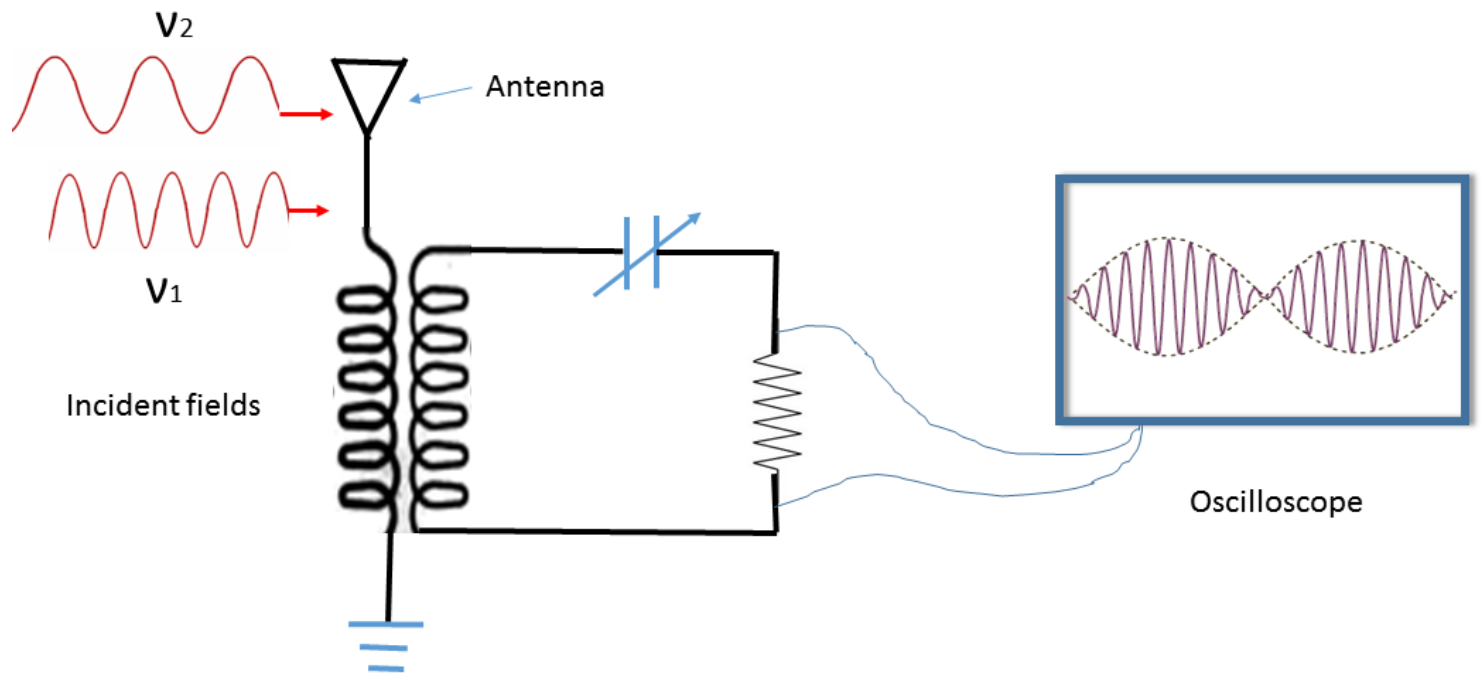

Figure 2. Two microwave fields of the same amplitude but slightly different frequencies are incident on an antenna. The conduction electrons respond to the superposition of the two electric fields. The induced current in the LCR circuit oscillates with mean a frequency $\left(v_{1}+v_{2}\right) / 2$ and beat frequency $\left(v_{1}-v_{2}\right) / 2$.

The induced current in the LRC circuit responds to electric fields in Eq. (3) and Eq. (4) by oscillating with a resonant frequency that closely matches the frequency of the superposed electric fields. It is a continuous interaction and exchange of energy. As long as the LRC circuit frequency is tuned to the frequency of the incoming microwave, the signal will be detected.

\section{VISISBLE WAVE DETECTORS}

The dipoles detecting visible light waves $(384-770 \mathrm{THz})$ also respond to the superposition of the EM waves received. For visible light, the interaction involves a transfer of quantum of energy of a given frequency that matches the allowed quantum energy levels of the detecting device. These energy exchanges are quadratic. It is proportional to the square of the amplitude of the electric field. Let us represent the incident electric fields as

$$
E=a \exp [-i 2 \pi v t]
$$

where $v$ is frequency in the visible range and $a$ is the amplitude of the electric field. If the visible light detector's dipole polarization is $\chi$, then the dipole response to the incident electric field may be represented by [8]

$$
\Psi=\chi E=\chi a \exp [i 2 \pi v t]
$$

If the dipole experiences simulations from two visible lights of the same amplitude but different frequencies that are close to each other $\psi_{1,2} \equiv \chi a \exp \left[-i 2 \pi v_{1,2} t\right]$, the detector absorbs energy proportional to the square modules of the stimulations by the two fields as represented by $D(t)$ in the equation below.

$$
D(t)=\Psi * \Psi=\left|\psi_{1}+\psi_{2}\right|^{2}=\left|\chi a e^{-i 2 \pi v_{1} t}+\chi a \mathrm{e}^{-i 2 \pi v_{2} t}\right|^{2}=2 \chi^{2} a^{2}\left[1+\cos 2 \pi\left(v_{1}-v_{2}\right) t\right]
$$

Unlike the AC current in the microwave LRC circuit, this generates a DC photo current in the detecting circuit. Depending on the response time of the detecting device, this current oscillation about the DC line may be observed. The detecting dipoles respond by absorbing EM energy that allows them to make the allowed quantum transition from the valence band 
to the conduction band of the semiconductor detecting device (see Figure 3.). For discrete energy levels of relatively free atoms, we have $\Delta E=h v$.

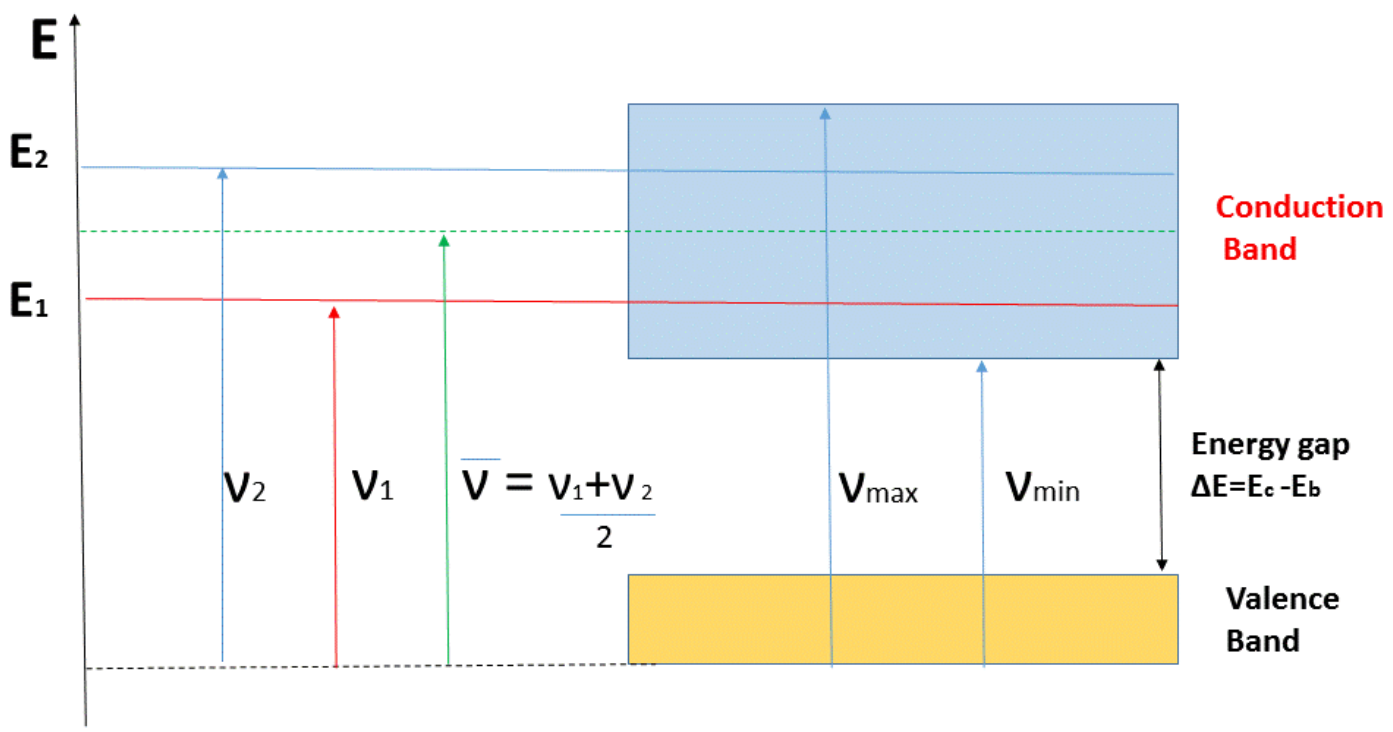

Figure 3. For a detector with discrete energy levels $E_{1}$ and $E_{2}$, it needs a quantum of energy $E=h v$ that corresponds to the frequency $v_{1}$ and $v_{2}$, respectively, to make the transition. For broad band energy levels, the electrons need to absorb the minimum gap energy needed to jump to the conduction band and form a detectable photocurrent. An EM field with mean frequency would have enough needed gap energy but this has not been observed to simulate the detector with discrete energy levels as the transition is a quadratic process [10].

In addition to the differences in the detected current behaviors in the microwave and visible light detectors, there is a difference in the frequency response to the difference $\left(v_{1}-v_{2}\right) / 2$ and the mean $\left(v_{1}+v_{2}\right) / 2$ frequencies that result from superposition of two incident EM waves of frequencies $v_{1}$ and $v_{2}$. The microwave detector responds to both the mean and difference frequencies. The visible detector responds to their difference as given by Eq. (7) resulting in a photocurrent that oscillates about a DC current $[6,10]$.

\section{CONCLUSION}

It is an important educational foundation in optics to point out and emphasize the difference in EM wave detection processes between low frequency radio and microwaves and the optical frequencies. The detectors in both the microwave and the visible frequencies are conduction electrons that interact and respond to the incident electric field. In the case of microwave, the electric field induces an AC current in the LRC circuit tuned to the frequency of the receiving antenna. There is a linear response by the conduction electrons proportional to the amplitude of incident superposed EM electric field amplitudes.

The detectors in the optical frequency range cannot continuously respond to the incident electric field because of the energy gap between the valance and conduction bands in the detectors. They only respond when the interaction between the dipole and the electric field simulates and transfers the gap energy $\Delta E=h v$ needed for the electron to jump from the valence band to the conduction band. Unlike the oscillating AC current in the LRC circuit in the microwave detector, the current in the visible detector is a one way DC current. A fast detector can demonstrate an oscillation about this DC current according to Eq. (7). There is a quadratic response by conduction electron in the photocurrent proportional to the square modules of the amplitude of the incident superposed EM fields. 


\section{REFERENCES}

[1] J.C. Maxwell, “A Dynamical Theory of the Electromagnetic Field”, Phil. Tran. , p.459-512, 1865.

[2] H. Hertz, [Electric Waves], Dover, New York, 1962.

[3] J.F. Mulligan, "Heinrich Hertz and the Development of Physics", Physics Today 42, 3, 50(1989).

[4] NASA website, https://www.jpl.nasa.gov/news/news.php?feature=7372, April 10, 2019.

[5] M. Ambroselli and C. Roychoudhuri, "Visualizing superposition process and appreciating the principle of non-interaction of waves," Proc. SPIE, Vol. 8121, 8121-1F (2011).

[6] M. Ambroselli, P. Poulos, C. Roychoudhuri, "Nature of EM waves as observed and reported by detectors for radio, visible, and gamma frequencies," Proc. SPIE, Vol. 8121,8121-17 (2011).

[7] E. Hecht and A.R. Ganesan, [Optics], Pearson, 4 ${ }^{\text {th }}$ edition, 2008.

[8] M. Ambroselli, Ph. D. Thesis, Ch.3, P.56.

[9] D.L. Singupa, T.K. Sarkar, "IEE Antennas and Propagation Magazine”, Vol. 45, Issue 2, 2003.

[10] D.-I Lee and C. Roychoudhuri, "Measurement properties of superposed light beams carrying different frequencies," Optics Express 11(8), 944-51(2003). 